\title{
Ablación endometrial histeroscópica como tratamiento definitivo para el sangrado uterino anormal
}

\author{
Verónica Chamy P. ${ }^{1,2}$, Javiera Berhó $F^{1}$, Benjamín Tudela S. ${ }^{1}$, Anibal Scarella C. ${ }^{1}$ \\ ${ }^{1}$ Facultad de Medicina, Universidad de Valparaíso, Chile. ${ }^{2}$ Hospital Naval Almirante Nef de Viña del Mar, Chile
}

\section{RESUMEN}

Introducción: El sangrado uterino anormal (SUA) afecta a un 19\% de las mujeres. Su tratamiento definitivo consiste en la histerectomía, sin embargo la ablación endometrial histeroscópica surge como un tratamiento menos invasivo, menos riesgoso y más rápido. Objetivo: Evaluar la efectividad de la ablación endometrial histeroscópica como tratamiento definitivo para el SUA. Método: Estudio de cohorte retrospectivo obtenido de base de datos de protocolos operatorios específicos del total de histeroscopías quirúrgicas realizadas en el Servicio de Ginecología del Hospital Naval Almirante Nef de Viña del Mar, entre enero de 2002 y diciembre de 2010. Resultados: De las 507 histeroscopías quirúrgicas realizadas, 230 fueron por SUA. Del total de pacientes sometidas a ablación endometrial histeroscópica, $27(11,7 \%)$ necesitaron un segundo procedimiento, de las cuales sólo $15(6,5 \%)$ fueron por persistencia de SUA. No se encontró asociación entre la necesidad de un segundo procedimiento por SUA con características propias de la paciente ni del procedimiento quirúrgico. Conclusión: La ablación endometrial histeroscópica es una opción terapéutica efectiva y segura para el tratamiento del SUA, con baja necesidad de segundo procedimiento por persistencia de éste y bajo riesgo de complicaciones. La necesidad de un segundo procedimiento por SUA no se asocia con características propias de la paciente ni del procedimiento quirúrgico.

\section{PALABRAS CLAVE: Sangrado uterino anormal, ablación endometrial histeroscópica, histerectomía}

\section{SUMMARY}

Introduction: Abnormal uterine bleeding (AUB) affects $19 \%$ of women. Definitive treatment is hysterectomy, however hysteroscopic endometrial ablation is emerging as a less invasive, less risky and faster treatment. Objective: To evaluate the effectiveness of hysteroscopic endometrial ablation as definitive treatment for AUB. Method: Retrospective cohort study database obtained from specific surgical protocols of all surgical hysteroscopies performed in the Gynecology Department of Hospital Naval Almirante Nef de Vina del Mar, between January 2002 and December 2010. Results: Of the 507 surgical hysteroscopies performed, 230 were because of AUB. Of all patients undergoing hysteroscopic endometrial ablation, $27(11.7 \%)$ required a second procedure, of which only $15(6.5 \%)$ were due to persistence of AUB. No association was found between the need for a second procedure because of AUB with patient characteristics or the surgical procedure itself. Conclusion: Hysteroscopic endometrial ablation is a safe and effective therapeutic option for AUB treatment, with a low need for a second procedure due to its persistence and with low risk of complications. The need of a second procedure because of AUB is not associated with patient characteristics or the surgical procedure itself.

KEYWORDS: Abnormal uterine bleeding, hysteroscopic endometrial ablation, hysterectomy 


\section{INTRODUCCIÓN}

El sangrado uterino anormal (SUA) es un problema que afecta al $19 \%$ de las mujeres (1) y alrededor de un $10 \%$ a $30 \%$ en edad reproductiva (2). Este puede ser de causa estructural, como pólipos endometriales, adenomiosis, leiomioma, malignidad e hiperplasia, o no estructural por coagulopatías, trastornos ovulatorios, trastornos endometriales e iatrogenicas $(3,4)$.

Hay diversos tratamientos para el manejo del SUA, y su elección depende de las ventajas y desventajas de cada uno de éstos, como también, de los riesgos asociados a las características particulares de cada paciente y sus expectativas. Entre los tratamientos médicos destaca el dispositivo intrauterino con levonorgestrel (DIU-LNG) como el más efectivo (5). Dentro de los tratamientos quirúrgicos la histerectomía constituye el tratamiento definitivo, mientras que la ablación endometrial histeroscópica reporta un $96,1 \%$ de eficacia, siendo una técnica menos invasiva (6).

La ablación endometrial histeroscópica es un procedimiento introducido alrededor de 20 años atrás que busca remover el endometrio, incluyendo su capa basal, para evitar su regeneración posterior, terminando con ello el SUA y la fertilidad. Existen distintas técnicas para realizar este procedimiento, como la ablacion endometrial guiada por histeroscopía o de primera generación, que incluye la resección endometrial transcervical, el uso de rollerball y ablación láser, que requieren de medios de distensión gaseosos o líquidos (4,7-11). Los métodos de segunda generación incluyen 5 métodos ablativos: balón térmico, hidrotermia, energía microondas, electrocirugía de radiofrecuencia, y crioterapia $(6,9)$.

El procedimiento presenta como principales ventajas el ser menos invasivo, con mayor facilidad quirúrgica, un reducido tiempo intra y postoperatorio (3), rápida recuperación, y menores complicaciones perioperatorias (5), como hemorragia, perforación, hiponatremia dilucional y embolia gaseosa (7).

Entre las complicaciones tardías de la ablación endometrial se encuentra la persistencia de tejido endometrial residual posterior al procedimiento, que se asocia a riesgo de embarazo con una frecuencia entre 0,2 a $0,6 \%$, y a cáncer, dado que el procedimiento tiende a enmascarar el SUA propio de la neoplasia, que sirve como advertencia para su diagnóstico. Otras complicaciones tardías son hematometra en 1 a $2 \%$, y síndrome de esterilización tubaria, asociado a un hematometra localizado en un cuerno uterino, con menstruación retrógrada (12).
Según los trabajos de Engelsen y cols (13) y Rosati y cols (6), la efectividad del procedimiento para evitar la histerectomía va desde un 83,4 a 95\% respectivamente, teniendo como principal determinante para el éxito del procedimiento la completa remoción del endometrio. Esto requiere de un endometrio relativamente delgado, como en el estado postmenopáusico o postmenstrual o posterior a la administración de tratamiento hormonal, a pesar de que otros estudios indican que el uso de la terapia hormonal previa al procedimiento no interferiría en el resultado de la ablación endometrial.

Las contraindicaciones para realizar una ablación endometrial histeroscópica incluyen embarazo, infección del tracto genital y carcinomas uterinos (7). También se describe en la literatura que la adenomiosis sería una causa importante de falla de la técnica, principalmente aquella que penetra más de 2,5 mm en el espesor miometrial, sin embargo no es considerada una contraindicación propiamente tal (10).

Es así como la ablación endometrial histeroscópica al ser un procedimiento menos invasivo y de menor costo, surge como una alternativa para mujeres que desean conservar su útero o en aquellas en que está contraindicada la cirugía mayor, como es la histerectomía.

El objetivo de esta comunicación es evaluar la efectividad de la ablación endometrial histeroscópica como tratamiento definitivo para el SUA.

\section{PACIENTES Y MÉTODO}

Estudio analítico retrospectivo obtenido de la base de datos de protocolos operatorios específicos de 507 histeroscopías quirúrgicas, realizadas en el Servicio de Ginecología del Hospital Naval Almirante Nef de Viña del Mar, entre enero de 2002 y diciembre de 2010, con al menos con un año de seguimiento.

Se incluyeron las histeroscopías quirúrgicas con ablación endometrial realizadas a pacientes con diagnóstico de SUA (polimenorrea, menorragia, metrorragia, menometrorragia y sangrado posmenopáusico) con fertilidad cumplida, en premenopausia o postmenopausia, con o sin terapia de reemplazo hormonal y con o sin tamoxifeno.

Se excluyeron las histeroscopías diagnósticas y por dispositivo intrauterino, las histeroscopías quirúrgicas por infertilidad y por anormalidades müllerianas, las realizadas por hallazgos ecográficos sospechosos de patología endocavitaria sin SUA y aquellas pacientes con histerometría $>12 \mathrm{~mm}$. Complementariamente se analizó la información de los datos registrados en las fichas clínicas por dos 
operadores independientes, además de un seguimiento ginecológico hasta diciembre de 2011.

Los procedimientos quirúrgicos fueron realizados por operador único con equipamiento marca Storz, que incluye resectoscopio de $9 \mathrm{~mm}$ con asa y roller, y óptica de $4 \mathrm{~mm}$ a 30 grados, energía monopolar electro conmed electrosurgery system 2500 (coagulación 50 y corte 70), cámara Endovision Telecam modelo 202120-20, con generador de luz fría Xenon Nova modelo 201315-20 y monitor de video Trinitron color pum-14n5a/14n5e/14n5u. Para distensión uterina se utilizo un histeromat, con presión intrauterina de $75 \mathrm{~mm} \mathrm{Hg}$ y volumen de infusión de $200 \mathrm{ml} /$ minuto, y aspiración de 0,25 bar. El medio de distensión utilizado fue glicina al 1,5\%.

Las variables analizadas fueron edad, estado hormonal (pre o postmenopausia), uso de terapia de reemplazo hormonal (TRH), uso de tamoxifeno, diagnóstico y grosor endometrial ecográfico preoperatorio, histerometría, volumen total de medio de distensión utilizado, volumen de medio de distensión perdido, volumen de medio de distensión perdido de riesgo ( $\geq 1000 \mathrm{ml}$ ), presencia y tipo de complicación intraoperatoria y postoperatoria, calidad de la ablación, diagnóstico histopatológico y presencia de adenomiosis en el material de ablación, necesidad, tipo y causa del segundo procedimiento, latencia entre la ablación y el segundo procedimiento, diagnóstico histopatológico y presencia de adenomiosis en la pieza operatoria.

Para el análisis estadístico las variables fueron almacenadas en una base de datos Microsoft Excel
2003 y analizadas en Stata 10.0. Todos los datos fueron sometidos a análisis descriptivo, para lo cual se utilizó distribución de frecuencias para variables cualitativas, media y desviación estándar para variables cuantitativas con distribución normal, y rangos intercuartiles y mediana para variables cuantitativas de distribución no normal. Se realizó análisis exploratorio con pruebas de hipótesis paramétricas y no paramétricas según correspondía. Se consideró como significativo un valor de $p \leq 0,05$.

\section{RESULTADOS}

Durante el periodo estudiado se realizaron 507 histeroscopías quirúrgicas, 571 histerectomías abdominales y 416 histerectomías vaginales. La cantidad y distribución de dichos procedimientos se presenta en Figura 1.

De 230 pacientes con SUA, la media de edad fue de 47,9 $\pm 8,9$ años (IC95\% 46,7 - 49,1), 78,2\% $(n=180)$ eran premenopáusicas y $21,7 \% \quad(n=50)$ postmenopáusicas. En las pacientes premenopáusicas la media de edad fue de 44,4 $\pm 4,5$ años (IC95\% 43,7 - 45,1) y en postmenopáusicas $60 \pm$ 9,6 años (IC95\% 57,8 - 63,3).

El uso de terapia de remplazo hormonal y de tamoxifeno fue consignado en 226 pacientes. EI $91,7 \%(n=207)$ de las pacientes no utilizaba terapia de remplazo hormonal y $8,3 \%(n=19)$ si la utilizaba. Solo el $3,1 \%$ ( $n=7)$ utilizaba tamoxifeno. La descripción de los parámetros anteriores según persistencia o no de SUA se muestra en la Tabla I.

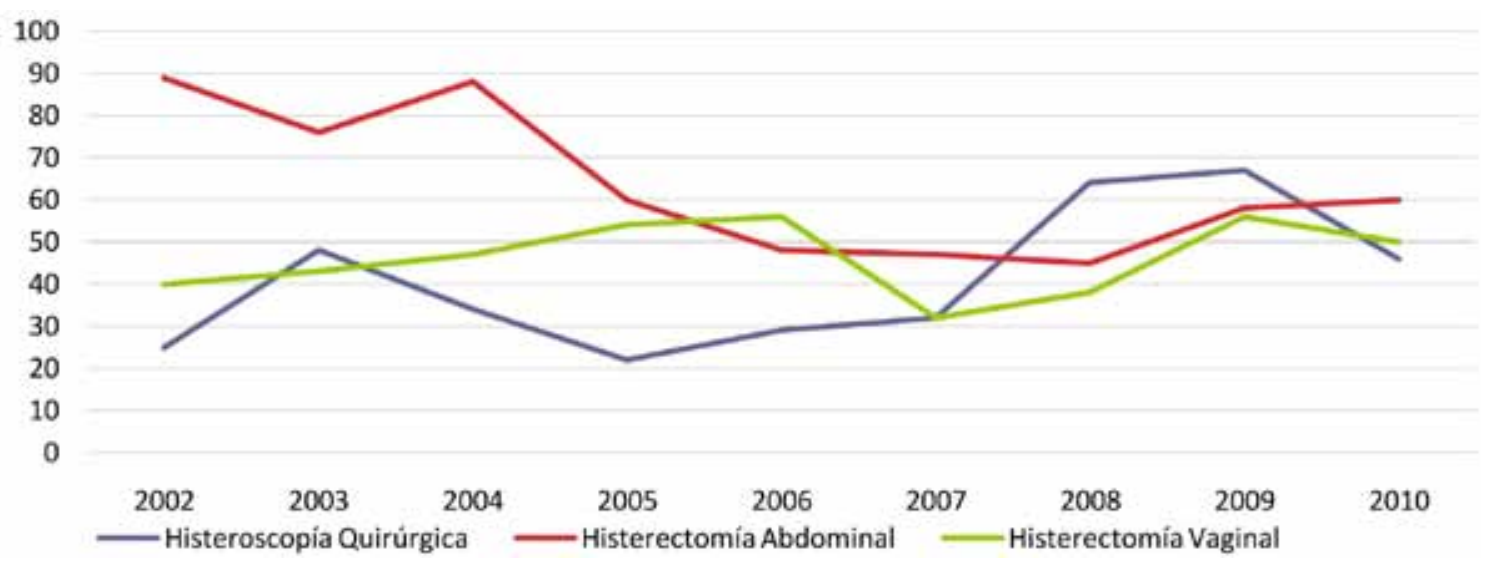

Figura 1. Histeroscopías quirúrgicas e histerectomías realizadas en el Hospital Naval Almirante Nef de Viña del Mar, enero de 2002 a diciembre de 2010. 


\section{Tabla I \\ CARACTERÍSTICAS GENERALES DE LAS PACIENTES SEGÚN PERSISTENCIA O NO DE SUA POSTERIOR A LA ABLACIÓN ENDOMETRIAL HISTEROSCÓPICA}

\begin{tabular}{|c|c|c|c|c|c|}
\hline \multirow[t]{2}{*}{ Parámetro } & \multicolumn{2}{|c|}{$\begin{array}{l}\text { Sin persistencia de SUA } \\
\qquad(n=215)\end{array}$} & \multicolumn{2}{|c|}{$\begin{array}{l}\text { Con persistencia de SUA } \\
\qquad(n=15)\end{array}$} & \multirow[t]{2}{*}{ Valor $p$} \\
\hline & Media \pm DS & IC95\% & Media \pm DS & IC95\% & \\
\hline $\begin{array}{l}\text { Edad (años) } \\
\text { Grosor endometrial (mm) } \\
\text { Histerometría }\end{array}$ & $\begin{aligned} 48,2 & \pm 9 \\
10,5 & \pm 5,8 \\
8,6 & \pm 1,2\end{aligned}$ & $\begin{array}{l}47-49,4 \\
9,4-11,6 \\
8,4-8,8\end{array}$ & $\begin{array}{l}44 \pm 6,7 \\
13 \pm 3,9 \\
8,2 \pm 1,3\end{array}$ & $\begin{array}{c}40,2-47,7 \\
9,9-16,0 \\
7,4-9,1\end{array}$ & $\begin{array}{l}0,07 \text { * } \\
0,2^{*} \\
0,34 \text { * }\end{array}$ \\
\hline $\begin{array}{l}\text { Estado menopáusico } \\
\text { premenopausia } \\
\text { postmenopausia }\end{array}$ & \multicolumn{2}{|c|}{$\begin{array}{c}77,2 \%(166) \\
22,7 \%(49)\end{array}$} & \multicolumn{2}{|c|}{$\begin{array}{c}93,3 \%(14) \\
6,6 \%(1)\end{array}$} & $\begin{array}{l}0,12 \dagger \\
0,12 \dagger\end{array}$ \\
\hline $\begin{array}{l}\mathrm{TRH} \\
\mathrm{No} \\
\mathrm{Si}\end{array}$ & \multicolumn{2}{|c|}{$\begin{array}{c}91,1 \%(195) \\
8,8 \%(19)\end{array}$} & \multicolumn{2}{|c|}{$\begin{array}{c}100 \%(12) \\
0 \%(0)\end{array}$} & $\begin{array}{l}0,26 \dagger \\
0,26 \dagger\end{array}$ \\
\hline $\begin{array}{l}\text { Tamoxifeno } \\
\text { No } \\
\text { Si }\end{array}$ & \multicolumn{2}{|c|}{$\begin{array}{c}96,6 \%(204) \\
3,3 \%(7)\end{array}$} & \multicolumn{2}{|c|}{$\begin{array}{c}100 \%(15) \\
0 \%(0)\end{array}$} & $\begin{array}{l}0,61 \dagger \\
0,61 \dagger\end{array}$ \\
\hline $\begin{array}{l}\text { Diagnóstico ecográfico pre } \\
\text { Endometrio normal } \\
\text { Endometrio engrosado } \\
\text { Tumor endocavitario § } \\
\text { Otros miomas \| } \\
\text { Adenomiosis }\end{array}$ & $\begin{array}{r}11 \\
23 \\
41 \\
17 \\
6\end{array}$ & $\begin{array}{l}(22) \\
(46) \\
(83) \\
(34) \\
(13)\end{array}$ & & & $\begin{array}{l}0,13 \neq \\
0,13 \neq \\
0,13 \neq \\
0,13 \neq \\
0,13 \neq\end{array}$ \\
\hline
\end{tabular}

* T student. † Fisher’s exact. ¥ Chi2. § Tumor endocavitario incluye pólipos endometriales y miomas submucosos. ॥ Otros miomas incluyen miomas intramurales y subserosos.

El diagnóstico ecográfico preoperatorio fue consignado en 212 casos, y la distribución de frecuencias se presenta en la Tabla II. El grosor endometrial ecográfico fue consignado en 120 casos, con una media de 10,6 $\pm 5,7 \mathrm{~mm}$ (IC95\% 9,6 - 11,7). En las pacientes premenopáusicas la media fue de $10,7 \pm 5,8 \mathrm{~mm}(\mathrm{IC} 95 \% 9,5-11,9)$ y en postmenopáusicas de 10,4 $\pm 5,2 \mathrm{~mm}$ (IC95\% 8,3-12,5).

La histerometría realizada previa a la ablación endometrial histeroscópica fue consignada en 196 casos, con una media de 8,6 $\pm 12 \mathrm{~cm}$ (IC95\% 8,4 $-87)$.

El volumen del medio de distensión utilizado durante el procedimiento fue consignado en 212 casos, con una media de $6230 \mathrm{ml}$ (rango: 4850 $9365 \mathrm{ml}$ ). La mediana del volumen del medio de distensión perdido en dichos casos fue de $400 \mathrm{ml}$ (rango: $200-750 \mathrm{ml})$ y el $21,2 \%(n=45)$ de las pacientes presentó un volumen de medio de distensión perdido $\geq 1000 \mathrm{ml}$.

La técnica quirúrgica fue consignada en 230 casos, siendo ablación endometrial completa en el $90 \%(n=207)$ e incompleta en el 10\% $(n=23)$.
Tabla II DIAGNÓSTICO ECOGRÁFICO PREOPERATORIO

\begin{tabular}{lcc}
\hline & Pacientes $(\mathrm{n}=212)$ & $\%$ \\
\hline Endometrio normal & 24 & 11,3 \\
Endometrio engrosado & 52 & 24,5 \\
Tumor endocavitario * $^{*}$ & 84 & 39,6 \\
Otros miomas $\dagger$ & 38 & 17,9 \\
Adenomiosis & 14 & 6,6
\end{tabular}

*Tumor endocavitario incluye pólipos endometriales y miomas submucosos. † Otros miomas incluyen miomas intramurales y subserosos

El $95,6 \%$ de las pacientes $(n=220)$ no presentó complicaciones intraoperatorias. El 4,3\% $(n=10)$ presentó complicaciones: $2(0,8 \%)$ perforaciones uterinas, $3(1,3 \%)$ hemorragias y $5(2,1 \%)$ desgarros cervicales. El 98,2\% $(n=225)$ no presentó complicaciones postoperatorias, mientras que el 1,7\% $(n=4)$ si presentó complicaciones: $2(0,8 \%)$ hiponatremias dilucionales, $1(0,4 \%)$ tromboembolismo pulmonar y $1(0,4 \%)$ paro cardiorrespiratorio. 
El diagnóstico del estudio histopatológico de la ablación endometrial histeroscópica fue consignada en 225 casos (Tabla III). El 49,3\% ( $n=111)$ de las pacientes presentó adenomiosis como resultado único o asociado a otros diagnósticos.

Del total de las pacientes sometidas a ablación endometrial histeroscópica, 27 (11,7\%) necesitaron un segundo procedimiento, de las cuales $15(6,5 \%)$ fueron por persistencia de SUA, y $12(5,2 \%)$ por otras causas, que se describen en la Tabla IV.

Los segundos procedimientos realizados fueron histerectomía vaginal en $10(4,3 \%)$ pacientes, histerectomía abdominal en $13(5,6 \%)$ e histeroscopía quirúrgica en $4(1,7 \%)$. La latencia al segundo procedimiento de las pacientes que lo requirieron fue de 5 meses (rango: $2-16$ meses).

El diagnóstico del estudio histopatológico de la pieza operatoria del segundo procedimiento fue consignada en 25 casos, y la distribución de frecuencia se presenta en la Tabla V. De las pacientes sometidas a segundo procedimiento $14(56 \%)$ presentaron adenomiosis como resultado único o asociado a otros diagnósticos.

Tabla III

RESULTADOS DEL ESTUDIO HISTOPATOLÓGICO DE LA ABLACIÓN ENDOMETRIAL HISTEROSCÓPICA

\begin{tabular}{lcc}
\hline & Pacientes $(\mathrm{n}=225)$ & $\%$ \\
\hline Tumor endocavitario & 115 & 51 \\
$\quad$ Pólipo endometrial & 88 & 39 \\
$\quad$ Mioma submucoso & 27 & 12 \\
Otros miomas * & 46 & 20,4 \\
Hiperplasia endometrial & 12 & 5,2 \\
$\quad$ Simple & 10 & 4,4 \\
$\quad$ Con atipias & 2 & 0,8 \\
Atrofia endometrial & 6 & 2,6 \\
Endometrio normal & 40 & 17,7 \\
Cáncer endometrial & 2 & 0,8 \\
Pólipo endometrial + hiperplasia con atipias & 3 & 1,3 \\
Pólipo endometrial + cáncer endometrial & 1 & 0,4 \\
\hline
\end{tabular}

* Otros miomas incluyen miomas intramurales y subserosos

Tabla IV

CAUSAS DE SEGUNDO PROCEDIMIENTO SEGÚN EL TOTAL DE PACIENTES

\begin{tabular}{lcc}
\hline & Pacientes $(\mathrm{n}=27)$ & $\%$ \\
\hline Persistencia de SUA & 15 & 6,5 \\
Prolapso & 1 & 0,4 \\
Cáncer endometrial o hiperplasia con atipias & 8 & 3,4 \\
Hallazgo ecográfico post-ablación & 2 & 0,8 \\
Algia pélvica & 1 & 0,4
\end{tabular}

SUA: sangrado uterino anormal. 


\section{Tabla V \\ RESULTADOS DEL ESTUDIO HISTOPATOLÓGICO DE LA PIEZA OPERATORIA}

\begin{tabular}{lcc}
\hline & Pacientes $(\mathrm{n}=25)$ & $\%$ \\
\hline Tumor endocavitario & 3 & 12 \\
$\quad$ Pólipo endometrial & 2 & 8 \\
$\quad$ Mioma submucoso & 1 & 4 \\
Otros miomas* $^{*}$ & 8 & 32 \\
Hiperplasia endometrial & 2 & 8 \\
$\quad$ Simple & 1 & 4 \\
$\quad$ Con atipias & 1 & 4 \\
Endometrio normal & 11 & 44 \\
Endometrio atrófico & 1 & 4
\end{tabular}

* Otros miomas incluyen miomas intramurales y subserosos.

Al comparar aquellas pacientes con necesidad de segundo procedimiento por persistencia de SUA, y las que no necesitaron un segundo procedimiento o lo necesitaron por otra causa, no hubo diferencias estadísticamente significativas respecto de: edad $(p=0,07)$, estado menopáusico $(p=0,12)$, uso de terapia de reemplazo hormonal $(p=0,26)$, uso de tamoxifeno $(p=0,61)$, diagnóstico ecográfico preoperatorio $(p=0,13)$, histerometría $(p=0,34)$, grosor endometrial $(p=0,2)$, volumen del medio de distensión utilizado $(p=0,47)$, pérdida de volumen medio $(p=68)$, pérdida $\geq 1000 \mathrm{ml}(p=0,45)$, técnica quirúrgica $(p=0,17)$, complicación intraoperatoria $(p=0,13)$ y postoperatoria $(p=0,23)$, tipo de complicación intraoperatoria $(p=0,15)$ y postoperatoria $(p=0,09)$, diagnóstico del estudio histopatológico de la ablación endometrial histeroscópica $(p=0,98)$ ni en presencia de adenomiosis $(p=0,52)$.

\section{DISCUSIÓN}

Durante el periodo de estudio, al comparar los procedimientos realizados considerando la incorporacion de la cirugía histeroscópica, se observa una tendencia a la disminución de las histerectomías especialmente abdominales, mientras que aumenta progresivamente el número de ablaciones endometriales histeroscopicas, constituyendo el 30 a $40 \%$ del total en el año 2010. Esto refleja un cambio en el manejo de las patologías uterinas benignas, prefiriéndose procedimientos menos invasivos, de técnica quirúrgica de menor complejidad, de menor tiempo intra y postoperatorio, y menor riesgo de complicaciones (6).
En los casos de este estudio no se realizó preparacion endometrial con análogos de $\mathrm{GnRH}$ previo a la ablación endometrial. A pesar de que se ha recomendado el uso de estos fármacos para aumentar el éxito del procedimiento, otros estudios no han demostrado diferencias significativas entre las pacientes tratadas y no tratadas previamente en cuanto a complicaciones ni necesidad de un segundo procedimiento (13).

Los tumores endocavitarios, incluyendo pólipos endometriales y miomas submucosos, son una de las principales causas de SUA (5). En este estudio los tumores endocavitarios asociados a sangrado uterino anormal fueron el principal diagnóstico ecográfico preoperatorio $(39,6 \%)$ y la mayor parte de los hallazgos del estudio histopatológico de la ablación endometrial histeroscópica (51\%), en donde el $39 \%$ correspondió a pólipos endometriales y $12 \%$ a miomas submucosos. Se ha descrito a los pólipos como factor de riesgo en el desarrollo de cáncer endometrial, encontrando en esta serie tres casos de pólipo con atipias y un caso de pólipo con cáncer, de un total de 92 casos con pólipos demostrados en el estudio histopatológico.

La evaluacion de las variables consideradas de riesgo de fracaso de la ablación endometrial para controlar el sangrado uterino, como edad, estado menopáusico, uso de terapia de reemplazo hormonal, uso de tamoxifeno, grosor endometrial, histerometría, la presencia de adenomiosis y diagnóstico ecográfico preoperatorio, no demostró diferencias estadísticamente significativas al comparar las pacientes que, posterior a la ablación, necesitaron un segundo procedimiento por persistencia de SUA, 
con aquellas que no lo necesitaron. Esto concuerda con los resultados obtenidos en otros estudios (6). Por lo tanto, estas características podrían no considerarse contraindicaciones del procedimiento.

La adenomiosis se presentó como resultado único o asociado a otro diagnóstico del estudio histopatológico de la ablación endometrial en un $49,3 \%$ de los casos, de los cuales $9 \%$ necesitaron un segundo procedimiento por SUA. Esta diferencia no fue estadísticamente significativa al comparar con aquellas pacientes que no necesitaron segundo procedimiento por SUA, en contraste con otros estudios que proponen a la adenomiosis como causa importante del fracaso de la ablación endometrial histeroscópica (10).

Se analizó el volumen de distensión perdido $\geq 1000 \mathrm{ml}$ como posible factor de riesgo para el fracaso de la ablación endometrial histeroscópica, de acuerdo a algunas publicaciones (5). Este estudio no mostró diferencias estadísticamente significativas respecto a la necesidad de segundo procedimiento por SUA y no fue factor predictivo del fracaso de la ablación endometrial histeroscópica.

Se ha descrito que el factor determinante más importante en el fracaso de la ablación endometrial histeroscópica es la técnica quirúrgica, es decir, si fue completa o incompleta la remoción del endometrio (13). En este estudio, en un $10 \%$ de los casos la remoción endometrial fue incompleta, sin embargo no se encontró una diferencia estadísticamente significativa en el fracaso de la ablación endometrial histeroscópica para el tratamiento del SUA, al comparar con aquellas pacientes con remoción endometrial completa.

La mayoría de las pacientes no presentaron complicaciones intraoperatorias $(95,6 \%)$ ni postoperatorias $(98,2 \%)$, lo que concuerda con el bajo riesgo de complicaciones descrito por la literatura $(6,7)$. Las principales complicaciones perioperatorias de este estudio coinciden con las de otros estudios (7), a excepción de una paciente que presentó tromboembolismo pulmonar y paro cardiorrespiratorio debido a una comorbilidad previa, correspondiente a una distrofia muscular.

Del total de las pacientes sometidas a ablación endometrial histeroscópica, $27(11,7 \%)$ necesitaron un segundo procedimiento, de las cuales $15(6,5 \%)$ fueron por persistencia de SUA, fundamentalmente en el primer año posterior al primer procedimiento, lo que se condice con los resultados de Engelsen $y$ cols (13).

Por lo tanto, el éxito de la ablación endometrial histeroscópica como tratamiento definitivo del SUA fue de un $93,5 \%$. Resultados concordantes con la literatura (6). Se destaca que la recurrencia del
SUA se presenta en el primer año de realizado el procedimiento.

\section{CONCLUSIONES}

La ablación endometrial transcervical histeroscópica, es una opción terapéutica segura, con una efectividad del $93,5 \%$ para el tratamiento del SUA, con baja necesidad de segundo procedimiento por persistencia de éste y bajo riesgo de complicaciones tanto intraoperatorias como postoperatorias. La necesidad de un segundo procedimiento por SUA no se asocia con características del procedimiento quirúrgico ni aquellas propias de las pacientes analizadas en este estudio, por lo que no debieran ser consideradas contraindicación de la ablación endometrial histeroscópica. Los factores de riesgo de fracaso de la ablación endometrial para controlar el SUA requieren de más estudios con buena metodología. En esta publicación ni la edad ni la adenomiosis fueron factores de riesgo de fracaso de la técnica. La recurrencia del SUA se presenta en el primer año de realizada la ablación.

\section{REFERENCIAS}

1. Bradley LD. Abnormal uterine bleeding. Nurse Pract 2005;30(10):38-42, 45-9; quiz 50-1.

2. Edris F, Vilos GA, Al-Mubarak A, Ettler HC, HollettCaines J, Abu-Rafea B, Resectoscopic surgery may be an alternative to hysterectomy in high-risk women with atypical endometrial hyperplasia. J Minim Invasive Gynecol 2007;14(1):68-73.

3. Hill DA. Abnormal uterine bleeding: avoid the rush to hysterectomy. J Fam Pract 2009;58(3):136-42.

4. Munro MG, Critchley HOD, Fraser IS, para el Grupo de Trabajo de Trastornos Menstruales FIGO. La clasificación FIGO de causas de sangrado uterino anormal en los años reproductivos. Revista del Climaterio 2011;15(85):9-17.

5. Istre O, Qviqstad E. Current treatment options for abnormal uterine bleeding: an evidence-based approach. Best Pract Res Clin Obstet Gynaecol 2007;21(6):905-13.

6. Rosati M, Vigone A, Capobianco F, Surico D, Amoruso $E$, Surico N. Long-term outcome of hysteroscopic endometrial ablation without endometrial preparation. Eur J Obstet Gynecol Reprod Biol 2008;138(2):222-5.

7. American College of Obstetricians and Gynecologists. Hysteroscopy. ACOG Technology Assessment in Obstetrics and Gynecology No. 4. Obstet Gynecol 2005;106:439-42.

8. Papadopoulos NP, Adam Magos A. First generation endometrial ablation: rollerball vs loop vs laser. Best Pract Res Clin Obstet Gynaecol 2007;21(6):915-29.

9. Nezhat C. Operative Gynecology Laparoscopic and Hysteroscopy. Third Edition. Cambridge, Cambrige University Press, 2008. pp 173-8. 
10. McCausland $\mathrm{V}$, McCausland $\mathrm{A}$. The response of adenomiosis to endometrial ablation/resection. Hum Reprod Update 1998;4(4):350-9.

11. Petrozza JC, Attaman JA. Hysteroscopy. eMedicine. com. Retrieved from http://emedicine.medscape.com/ article/267021-overview (Updated 2010,Jan 7)
12. Cooper JM, Brady RM. Late complications of operative hysteroscopy. Obstet Gynecol Clin North Am 2000;27(2):367-74.

13. Engelsen B, Woie K, Hordnes K. Transcervical endometrial resection: long-term results of 390 procedures. Acta Obstet Gynecol Scand 2006;85(1):82-7. 